Arq. Bras. Med. Vet. Zootec., v.68, n.1, p.141-146, 2016

\title{
Mensurações do bulbo ocular e cálculo do poder dióptrico da lente intraocular em miniporcos
}

\author{
[Ocular bulb measurements and diopter power calculation \\ of the lens in minipigs] \\ R. Barros, A.C.L. Rodrigues, U.C. Guberman, M.G. Gandolfi, N.B. Merlini, C.R. Teixeira, \\ C.R. Padovani, B.B. Brancalion, J.J.T. Ranzani, C.V.S. Brandão \\ Universidade Estadual Paulista - Unesp - Campus Botucatu, SP
}

\begin{abstract}
RESUMO
Objetivou-se com este trabalho determinar o poder dióptrico da lente intraocular (LIO) em miniporco e as dimensões do bulbo do olho. Foram utilizados 17 miniporcos, sadios, adultos, machos e fêmeas, com peso médio de $70 \mathrm{~kg}$. Em todos os olhos foram realizadas a ultrassonografia modo A, a ceratometria e a medida da distância limbo a limbo. O cálculo do poder dióptrico da LIO foi obtido utilizando-se as fórmulas Haigis, Hoffer Q, SRK/T, Holladay I e Holladay II e o software Holladay IOL Consultant ${ }^{\circledR}$. Na comparação entre o sexo e a lateralidade do olho, não houve diferença nas variáveis biométricas e poder da LIO. A aplicação das fórmulas (Haigis, Holladay II, Holladay I, SRK/T e Hoffer Q) possibilitou o cálculo do poder da LIO. A Holladay II, fórmula que melhor individualiza o bulbo do olho do miniporco, estima valor dióptrico ao redor de $41 \mathrm{D}$. Os miniporcos têm potencial como modelo experimental em oftalmologia, relacionado ao seu menor porte e à facilidade no manejo, especialmente em experimentos de longa duração.
\end{abstract}

Palavras-chave: biometria, catarata, ceratometria, LIO, miniporco

\begin{abstract}
The aim of this study was to determine the refractive power of intraocular lens (IOL) of mini pigs and the dimensions of the eyeball. A total of 17 (34 eyes) healthy, adult, males and female animals, with average weight of $70 \mathrm{~kg}$ were used. For every eye, A-mode ultrasound, keratometry and the measurement of limboto-limbo distance were conducted, all variables for calculating the refractive power of the IOL. The value was obtained using different formulas and Holladay IOL Consultant ${ }^{\circledR}$ Software. Additionally, the ocular measurements were compared per sex, laterality of the eye and the different formulas used in this study (Haigis, Hoffer Q, SRK / T, Holladay I and Holladay II). In the comparison between sex and laterality of the eye, there was no difference in biometric variables and power of the IOL. The application of the employed formulas (Haigis, Holladay II, Holladay I, SRK / T and Hoffer Q) allowed the IOL power calculation for this specie, and the observed value ranged between $39.58 \pm 2.15$ and $46.60 \pm 2.81$ diopters. Mini pigs play an important and growing role as an experimental model for study and practice of ophthalmic procedures, specially related to their smaller size and easy management in long-term experiments.
\end{abstract}

Keywords: biometry, cataract, IOL, keratometry, mini pig

\section{INTRODUÇÃO}

Os suínos são espécie de destaque em estudos biomédicos, porém o emprego de animais de produção para pesquisa é limitado, em virtude do

Recebido em 3 de junho de 2015

Aceito em 17 de setembro de 2015

E-mail: valeriasb@fmvz.unesp.br porte e da propensão ao ganho de peso, sendo cada vez mais proposto o uso do miniporco. Este possui, como vantagens, menor tamanho, consequente facilidade no manuseio, redução no dispêndio com alimentação, criação e, adicionalmente, possibilita estudos de longa duração (Kohn, 2012). 
Suas características biológicas gerais são similares às do porco, o qual é considerado modelo experimental consagrado, porém o grande diferencial do miniporco é o peso final na fase adulta, o qual se mantém entre 70 e $90 \mathrm{~kg}$ (Swindle, 2007).

A catarata é uma afecção frequente na oftalmologia, sendo definida como opacidade da lente ou cápsula lenticular, decorrente de alterações da arquitetura lamelar (Davidson e Nelms, 2007). O seu tratamento é eminentemente cirúrgico e implica a remoção da lente opacificada (facectomia).

No entanto, o olho torna-se afácico e altamente hipermetrope após a facectomia (Gaiddon et al., 1991; Liu et al., 2013). A correção dessa afacia é feita utilizando-se o implante de lentes intraoculares (LIO) sintéticas, cujo emprego é consagrado na medicina (Day et al., 2012; Eom et al., 2013a).

$\mathrm{Na}$ oftalmologia veterinária, há crescente preocupação em promover uma visão mais próxima possível à emetropia, objetivando melhora na qualidade de vida dos animais (Monteiro e Allemann, 2001; Harrington et al., 2013). O cálculo do poder dióptrico da LIO nas diferentes espécies ainda é assunto pouco explorado, portanto carente de informações estabelecidas (McMullen e Gilger, 2006).

Para a obtenção de resultado refrativo desejado, a biometria ocular é essencial, sendo a ultrassonografia modo A um método frequentemente utilizado (Kanski e Bowling, 2011). Várias fórmulas denominadas de terceira geração (Hoffer Q, Holladay I e SRK/T) foram desenvolvidas, levando em consideração a curvatura corneal e o comprimento axial do olho. Outras fórmulas mais recentes (Haigis e Holladay II) adicionaram a profundidade da câmara anterior, o diâmetro limbo a limbo e a espessura da lente, objetivando aperfeiçoar a exatidão da previsibilidade (Aristodemou et al., 2011).

A catarata secundária, ou opacidade de cápsula posterior (OCP), é uma complicação tardia da cirurgia de catarata e ocorre nos homens e nos animais. Dentre as manobras que visam minimizar seus efeitos prejudiciais na visão, destacam-se a implantação da LIO e sua concepção quanto a desenhos e formatos diferentes (Yuen et al., 2006). Estudos dessa natureza implicam experimentos de longo acompanhamento pós-operatório (Liu et al., 2013).

O crescente uso dos porcos em treinamentos médicos, os aspectos éticos envolvidos com a utilização especialmente de cães na experimentação animal estimularam o desenvolvimento deste estudo em miniporcos. Trata-se de um modelo interessante para experimento biomédico, colocando-os em destaque em relação a outros mamíferos, devido a fatores importantes a serem considerados referentes a manejo, reprodução e ética (Swindle, 2007).

Em razão da ausência de trabalhos na literatura consultada que apresentem valores de referência do bulbo do olho em miniporcos, este trabalho teve por objetivo avaliar a biometria do bulbo ocular e o poder dióptrico da LIO nessa espécie. Adicionalmente, buscou comparar se há diferença nessas variáveis considerando-se a lateralidade do olho e o sexo dos animais.

\section{MATERIAL E MÉTODOS}

Os procedimentos experimentais foram aprovados pela Câmara de Ética em Experimentação Animal da FMVZ - Unesp Botucatu (Processo n ${ }^{\circ}$ 91/2013) e seguiram as normas da "Association for Research in Vision and Ophthalmology" (ARVO).

Foram utilizados 17 miniporcos (Sus scrofa domestica), machos $(n=6)$ e fêmeas $(n=11)$, adultos, com idade entre seis meses e um ano e peso médio de $70 \mathrm{~kg}$.

Foram selecionados animais clinicamente saudáveis, após terem sido submetidos ao exame clínico e oftalmológico, com auxílio de biomicroscopia em lâmpada de fenda (SL-15, Kowa ${ }^{\circledR}$, Japão), oftalmoscopia binocular indireta (Lente OI-20 D, Ocular Instruments inc, USA), tonômetro de aplanação (Reichertß, USA) e teste lacrimal de Schirmer.

Todos os olhos $(n=34)$ foram avaliados, constituindo-se um único grupo experimental, e submetidos aos exames necessários para $\mathrm{o}$ cálculo do poder dióptrico da lente intraocular: 
diâmetro horizontal corneal, distância limbo a limbo do olho (Compasso cirúrgico Castroviejo, PrevTech $\AA$, Brasil); ceratometria (realizadas três aferições consecutivas em cada olho, e medidos $\mathrm{K} 1$ : valor do menor meridiano da córnea; K2: valor do maior meridiano da córnea; $\mathrm{K}$ : média de K1 e K2.) (SP-100, Tomey®, Japão); ultrassonografia modo A (comprimento axial do bulbo ocular, câmara anterior, espessura da lente e câmara vítrea) (AL-100, Tomey®, Japão).

Para obtenção do poder dióptrico da lente, foi utilizado software Holladay IOL Consultant (HicSoap ${ }^{\circledR}$, USA) para as fórmulas Holladay II, Holladay I, Hoffer Q e SRK/T; a fórmula Haigis foi calculada utilizando-se o software do aparelho de ultrassonografia modo A.

Para análise comparativa das variáveis biométricas, considerando-se a lateralidade do olho e a diferença entre o sexo, foi utilizado o teste $\mathrm{t}$ de Student para amostras dependentes e independentes, respectivamente (Zar, 1999).

Na comparação do poder dióptrico da lente, calculado por diferentes fórmulas, foi utilizada a técnica da análise de variância multivariada para o modelo de medidas repetidas, complementada com o teste de comparações múltiplas de Bonferroni (Johnson e Wichern, 1998).

A análise dos valores do poder dióptrico, considerando-se a fórmula Holladay II como padrão ouro, foi realizada utilizando-se o modelo de regressão linear (Draper e Smith, 1998). O nível de significância considerado foi de 5\% para todos os testes (Zar, 1999).

\section{RESULTADOS E DISCUSSÃO}

Como características positivas, podem-se ressaltar o fácil manejo e a alta taxa reprodutiva, a qual permitiu ter o número de animais adequado para o desenvolvimento do experimento e para a análise estatística.

Poucos trabalhos com mensurações oftalmológicas no miniporco foram encontrados na literatura consultada. Neste estudo, não houve diferença significativa no teste lacrimal de Schirmer (TLS), na mensuração da pressão intraocular (PIO), no diâmetro corneal e nas variáveis biométricas ultrassonográficas modo $\mathrm{A}$, na comparação entre lateralidade do olho e entre machos e fêmeas.

A média geral obtida foi de $8,12 \pm 2,65 \mathrm{~mm} / \mathrm{min}$ no teste lacrimal de Schirmer, $17,92 \pm 4,81 \mathrm{mmHg}$ na PIO e $14,10 \pm 0,85 \mathrm{~mm}$ no diâmetro corneal. Os primeiros são inferiores aos descritos para porcos, cuja produção lacrimal foi de $15,6 \pm 3,7 \mathrm{~mm} / \mathrm{min}$ (Trbolova e Ghaffari, 2012), bem como para outros animais domésticos, excetuando-se os coelhos (Ollivier et al., 2007).

Ressalta-se que, para o miniporco especificamente, não foram encontrados valores de referência para o TLS. Quanto à PIO, na maioria dos animais, esta varia entre 15 e 25 mmHg (Ollivier et al., 2007), resultado que corrobora os achados do presente estudo $(17,92 \mathrm{mmHg})$.

A média geral encontrada para biometria ocular foi comprimento axial de $18,30 \pm 0,48 \mathrm{~mm}$; câmara anterior de $3,12 \pm 0,38 \mathrm{~mm}$; espessura da lente de $5,88 \pm 0,34 \mathrm{~mm}$ e câmara vítrea de $9,39 \pm 0,55 \mathrm{~mm}$. A distância limbo a limbo horizontal observada foi de $14,10 \pm 0,85 \mathrm{~mm}$. Nielsen e Lind (2005) relataram uma média de $19 \mathrm{~mm}$ para comprimento axial e poder corneal de 44,10 $\pm 1,50$ D em 30 miniporcos Gottingen, valores similares aos observados no presente estudo.

Em comparação às outras espécies domésticas, encontrou-se comprimento axial de $39,23 \pm 1,26 \mathrm{~mm}$ em cavalos (McMullen e Gilger, 2006) e 20,91 $\pm 0,53 \mathrm{~mm}$ em felinos (Gilger et al., 1998) e 20,43 $\pm 1,48 \mathrm{~mm}$ em cães (Gaiddon et al., 1991).

Quanto à ceratometria, não houve diferença significativa na lateralidade do olho, porém as fêmeas $(43,84 \pm 2,10$ dioptrias $)$ apresentaram valores dióptricos maiores da curvatura corneal em relação aos machos (41,90 $\pm 0,93$ dioptrias). A média geral da ceratometria foi de $42,10 \pm 2,19$ dioptrias (D) para $\mathrm{K} 1 ; 44,29 \pm 1,94 \mathrm{D}$ para $\mathrm{K} 2$; $43,15 \pm 2,00 \mathrm{D}$ para K.

A realização dos exames de ceratometria e ultrassonografia modo A nos miniporcos foram factíveis, porém, devido à agitação e à conformação anatômica desses animais, não foi possível realizar a ultrassonografia modo A pelo 
método de imersão, apesar de sua vantagem em relação ao método de contato (Holladay, 1997).

Optou-se pela ultrassonografia modo A, pois os valores obtidos na mensuração apresentam os melhores resultados, de forma geral, quanto à ultrassonografia modo B (Dietrich, 2007).

Outro método mais preciso é o biômetro óptico de não contato, que utiliza um feixe de luz para obter as medidas, porém ainda não acessível para a área da medicina veterinária, pois precisa da colaboração do paciente (Monteiro e Allermann, 2001). Oliveira et al. (2004) descreveram que, apesar das vantagens e da tecnologia do biômetro óptico em relação ao ultrassônico modo $\mathrm{A}$, não observaram diferença estatística entre os aparelhos em humanos.

A utilização de miniporcos machos e fêmeas e a avaliação realizada nos dois olhos permitiram o estudo de possíveis variabilidades dos resultados quanto ao sexo e à lateralidade, o que não foi encontrado, corroborando os achados de Gaiddon et al. (1991), Montiani-Ferreira et al. (2008) e Toni et al. (2010).

No estudo comparativo do poder dióptrico da lente intraocular calculado, utilizando-se diferentes fórmulas (Haigis Standard, SRK/T, Hoffer Q, Holladay I e Holladay II), notou-se diferença significativa $(\mathrm{P}<0,001)$. Entretanto, quanto à lateralidade do olho e ao sexo, não houve diferença estatística $(\mathrm{P} \geq 0,05)$.
As fórmulas SRK/T, Hoffer Q e Holladay foram incluídas neste trabalho, considerando-se que ainda são amplamente utilizadas e estudadas (Aristodemou et al., 2011; Eom et al., 2013b; Carifi et al., 2015). Estas necessitam apenas do comprimento axial do olho e do poder corneal para se obter a previsibilidade da LIO (Narvaéz et al., 2006; Aristodemou et al., 2011).

Outras fórmulas desenvolvidas posteriormente, como a Holladay II e a Haigis, pressupõem o uso de variáveis adicionais, como diâmetro corneal limbo a limbo, comprimento de câmara anterior e da lente. A principal diferença entre essas fórmulas e as de terceira geração é que as últimas predeterminam a posição efetiva da lente e consideram que todos os olhos apresentam uma proporção linear entre comprimento axial e comprimento de câmara anterior (Day et al., 2012).

Os olhos do miniporco são classificados como curtos na oftalmologia médica, considerando-se os limites estabelecidos entre $18,00 \mathrm{~mm}$ e $22,00 \mathrm{~mm}$. Sabe-se que, em olhos curtos e extremamente curtos, essa proporção nem sempre é obedecida, o que aumenta a chance de erro refrativo final no cálculo (Holladay et al., 1995; Carifi et al., 2015), remetendo, portanto, aos olhos dos miniporcos. As fórmulas mais recentes minimizam esse erro devido às mensurações adicionais (Narvaéz et al., 2006; Eom et al., 2013a).

O poder dióptico médio da lente obtido pelas diferentes fórmulas está apresentado na Tab. 1.

Tabela 1. Poder dióptrico médio calculado da lente intraocular, em dioptrias (D), segundo as diferentes fórmulas, em miniporcos

\begin{tabular}{lccc}
\hline Fórmulas & Média & Valor mínimo & Valor máximo \\
\hline Haigis & $46,6 \pm 2,81 \mathrm{e}$ & 38,05 & 50,75 \\
SRK/T & $39,58 \pm 2,15 \mathrm{a}$ & 33,51 & 43,05 \\
Hoffer Q & $43,09 \pm 2,8 \mathrm{~d}$ & 35,49 & 48,01 \\
Holladay I & $41,08 \pm 2,38 \mathrm{~b}$ & 34,42 & 44,82 \\
Holladay II & $41,55 \pm 2,31 \mathrm{c}$ & 34,6 & 45,21 \\
\hline
\end{tabular}

Letras minúsculas diferentes indicam diferença significativa, $\mathrm{P} \leq 0,001$.

A fórmula SRK/T (Tab. 1) resultou na menor dioptria para o miniporco, enquanto a formula Haigis foi a que estipulou a maior dioptria. Tendo como base (padrão ouro) a fórmula Holladay II, verificou-se que a SRK/T subestima os valores em 5,11\%; as fórmulas Hagis, Hoffer Q e Holladay I superestimam em 12,18\%; 3,75\% e $1,46 \%$, respectivamente. 
A fórmula Holladay II foi selecionada neste estudo como padrão ouro por ser a que utiliza maior número de variáveis e individualização do olho, como já relatado anteriormente, minimizando possíveis erros relacionados às proporções do olho curto.

Notaram-se, no estudo de regressão, menores variações quando comparadas as fórmulas Holladay I e Hoffer Q, sugerindo que, especialmente a primeira, seria uma opção factível de terceira geração para o cálculo.

A aplicação prática do poder dióptrico calculado deve ser estimulada associada à avaliação de erro refrativo pós-operatório; diante do maior número de variáveis diretas, acredita-se que a dioptria estimada pela Holladay II seria um ponto de partida interessante.

Narvaéz et al. (2006) testaram as fórmulas Hoffer Q, Holladay I, Holladay II e SRK/T quanto a sua previsibilidade do poder dióptrico da LIO para olhos curtos até extremamente longos em humanos e não observaram diferença significativa no cálculo das fórmulas.

Eom et al. (2013a) afirmaram que, em olhos curtos, não ocorre diferença significativa entre as fórmulas Hoffer Q e Haigis. Porém, à medida que o comprimento da câmara anterior fica menor que $2,40 \mathrm{~mm}$, começa a ocorrer diferença no resultado final entre as fórmulas. Neste estudo com miniporcos, a diferença de 3D foi observada, mesmo com valores de câmara anterior superior a $2,40 \mathrm{~mm}$.

Cada espécie animal possui diferenças anatômicas oculares e evolutivas que determinam esse poder refrativo da lente (Gilger et al., 2004); o conhecimento desse poder refrativo nos miniporcos tem sido pouco relatado.

Esta variável é fundamental para a seleção da LIO a ser implantada, para a realização de testes futuros e de estudos experimentais com tipos, formatos e tratamento superficial da LIO, visando à redução da opacidade de cápsula posterior, que exigem maior acompanhamento pós-operatório.

Novas pesquisas devem ser estimuladas, objetivando o implante de lentes e a avaliação do poder refrativo efetivo no pós-cirúrgico. Dessa maneira, será possível comprovar a real dioptria da LIO a ser implantada no miniporco.

\section{CONCLUSÃO}

$\mathrm{Na}$ comparação entre o sexo e a lateralidade do olho, não há diferença nas variáveis biométricas, clínicas oftalmológicas e poder da lente intraocular. O bulbo do olho do miniporco, segundo a ultrassonografia modo A e os critérios estabelecidos na oftalmologia médica, é classificado como olho curto. A aplicação das fórmulas empregadas possibilita o cálculo do poder da lente intraocular do miniporco. A Holladay II, fórmula que melhor individualiza o bulbo do olho, estima valor dióptrico ao redor de 41D para o miniporco. Os miniporcos têm potencial como modelo experimental em oftalmologia, relacionado ao seu menor porte, à facilidade no manejo e ao baixo custo no uso de materiais, fármacos e alimentos.

\section{AGRADECIMENTOS}

Ao CNPq e à CAPES, pelo apoio financeiro.

\section{REFERÊNCIAS}

ARISTODEMOU, P.; CARTWRIGHT, N.E.K.; SPARROW, J.M.; JOHNSTON, R.L. Formula choice: Hoffer Q, Holladay 1 or SRK/T and refractive outcomes in 8108 eyes after cataract surgery with biometry by partial coherence interferometry. $J$. Cataract Refract. Surg., v.37, p.63-71, 2011.

CARIFI, G.; AIELLO, F.; ZYGOURA, V. et al. Accuracy of the refractive prediction determined by multiple currently available intraocular lens power calculation formulas in small eyes. Am. J. Ophthalmol., v.159, p.577-583, 2015.

DAVIDSON, M.G; NELMS, S.R. Diseases of the canine lens and cataract Formation. In: GELATT, K.N. (Ed). Veterinary ophthalmology. 4.ed. Florida: Blackwell Publishing, 2007. p.859-887.

DAY, A.C.; FOSTER, P.J.; STEVENS, J.D. Accuracy of intraocular lens power calculations in eyes with axial length $<22.00 \mathrm{~mm}$. Clin. Exp. Ophthalmol., v.40, p.855-862, 2012.

DIETRICH, U.M. Ophthalmic examination and diagnostics. In: GELATT, K.N. (Ed). Veterinary ophthalmology. 4.ed. Florida: Blackwell Publishing, 2007. p.507-519. 
DRAPER, N.R.; SMITH, H. (Eds.). Applied regression analysis. 3.ed. New York: John Wiley \& Sons, 1998. 736p.

EOM, Y.; KANG, S-Y.; SONG, J.S. et al. Comparison of Hoffer $\mathrm{Q}$ and Haigis formulae for intraocular lens power calculation according to the anterior chamber depth in short eyes. Am. J. Ophthalmol., v.157, p.818-824, $2013 \mathrm{a}$.

EOM, Y.; KANG, S-Y.; SONG, J.S. et al. Use of corneal power-specific constants to improve the accuracy of the SRK/T formula. Am. J. Ophthalmol., v. 120, p.477-481, $2013 \mathrm{~b}$.

GAIDDON, J.; ROSOLEN, S.G.; COOK, C.S.; PEIFFER JUNIOR, R. Use of biometry and keratometry for determining optimal power for intraocular lens implant in dogs. Am. J. Vet. Res., v.52, p.781-783, 1991.

GILGER, B.C.; WHITLEY, R.D.; McLAUGHLIN, S.A. et al. Clinicopathologic findings after experimental pmplantation of syntetic intraocular lenses in dogs. Am. J. Vet. Res., v.4, p.616-621, 2004.

GILGER, B.C.; DAVIDSON, M.G.; HOWARD, P.B.; Keratometry, ultrasonic biometry, and prediction of intraocular lens power in the feline eye. Am. J. Vet. Res., v.59, p.131-134, 1998.

HARRINGTON, J.T.; McMULLEN, R.J.; CLODE, A.B.; GILGER, B.C. Phacoemulsification and +14 diopter intraocular lens placement in a saddlebred foal. Vet. Ophthalmol., v.16, p.140-148, 2013.

HOLLADAY, J.T. Standardizing constants for ultrasonic biometry, keratometry, and intraocular lens power calculations. J. Cataract Refract Surg., v.23, p.1356-1370, 1997.

HOLLADAY, J.T.; GILLS, J.P.; LEIDLEIN, J.; CHERCHIO, M. Achieving emmetropia in extremely short eyes with two piggyback posterior chamber intraocular lenses. Ophthalmology, v.103, p.11181123, 1995.

JOHNSON, R.A.; WICHERN, D.W. (Eds.). Applied multivariate statistical analysis. 4.ed. Upper Saddle River: Prentice Hall, 1998. 816p.

KANSKI, J.J.; BOWLING, B. (Ed). Clinical ophthalmology: a systematic approach. 7.ed. Phyladelphia: Elsevier, 2011. 920p.

$\mathrm{KOHN}$, F. History and development of miniature, micro and minipigs. In: McANULTY, P.A.; DAYAN, A.D.; GANDERUP, N.C.; HASTINGS, K.L. (Eds), The minipig in biomedical research. Boca Raton, FL: CRC Press, 2012. p.3-16.
LIU, Y.C.; WONG, T.T.; MEHTA, J.S. Intraocular lens as a drug delivery reservoir. Curr. Opin. Ophthalmol., v.24, p.54-59, 2013.

McMUlLEN, R.J.; GILGER, B.C. Keratometry, biometry and prediction of intraocular lens power in the equine eye. Vet. Ophthalmol., v.9, p.357-360, 2006.

MONTEIRO, E.L.; ALLEMANN, N. Biometria óptica. Arq. Bras. Oftalmol., v.64, p.367-370, 2001.

MONTIANI-FERREIRA, F.; TRUPPEL, J.; TRAMONTIN, M.H. et al. The capybara eye: clinical tests, anatomic and biometric features. Vet. Ophthalmol., v.11, p.386-384, 2008.

NARVAÉZ，J.; ZIMMERMAN, G.; STULTING, R.D.; CHANG, D.H. Accuracy of intraocular lens power prediction using the Hoffer Q, Holladay 1, Holladay 2, and SRK/T formulas. J. Cataract Refract. Surg., v.32, p.2050-2053, 2006.

NIELSEN, L.S.; LIND, N.M. Measurements of three ocular parameters in the Gottingen minipig. Scand $J$. Lab. Anim. Sci., v.32, p.9-16, 2005.

OLIVEIRA, F.; MUCCIOLI, C.; LOPES, Y.C. et al. Biometrias óptica e ultra-sônica: comparação dos métodos usados para o calculo da lente intra-ocular acomodativa. Arq. Bras. Oftalmol., v.67, p.887-891, 2004.

OLLIVIER, F. J.; PLUMMER, C. E.; BARRIE, K. P. The eye examination and diagnostic procedures. In: GELATT, K. N. (Ed.). Veterinary ophthalmology. 4.ed. Iwoa: Blackwell, 2007. p.438-483.

SWINDLE, M.M. (Ed.). Swine in the laboratory. 2.ed. Boca Raton, FL: CRC Press, 2007. 471p.

TONI, M.C.; MEIRELLES, A.E.W.B.; GAVA, F.N. et al. Rabbits' eye globe sonographic biometry. Vet. Ophthalmol., v.13, p.384-386, 2010.

TRBOLOVA, A; GHAFFARI, M.S. Reference values for Schirmer tear tests I and II in clinically normal pigs. Vet. Ophthalmol., v.15, p.180-182, 2012.

YUEN, C.; WILLIAMS, R.; BATTERBURY, M.; GRIERSON, I. Modification of the surface properties of a lens material to influence posterior capsular opacification. Clin. Exp. Ophthalmol., v.34, p.568574, 2006.

ZAR, J.H. Biostatistical analysis 4.ed. New Jersey: Prentice Hall. 1999. 929p. 